\title{
Theory and Experiment of High-Speed Cross-Gain Modulation in Semiconductor Lasers
}

\author{
X. Jin, T. Keating, and S. L. Chuang
}

\begin{abstract}
We present theory and experiment for the high-speed modulation response of a quantum-well $(\mathrm{QW})$ laser in the presence of an external microwave modulated optical pump in the gain region. The model includes the effects of pump-induced stimulated recombination and cross-gain saturation. Expressions for the small-signal modulation response of the test laser under gain modulation are derived. We also present experimental results using a multiple-QW InGaAlAs Fabry-Perot (FP) laser at 1.552 $\mu \mathrm{m}$ as the test laser and an external pump by a $1.542 \mu \mathrm{m}$ DFB laser. Comparison between electrical modulation and optical cross-gain modulation (XGM) of the test laser is also presented, which shows improvement of the modulation bandwidth by optical XGM. Our data show a reduction of carrier lifetime with increasing optical pumping, a shift of the test-laser threshold current, a change in the $K$ factor, and a variation of the relaxation frequency with different pump powers. The experimental results agree very well with the theoretical results.
\end{abstract}

Index Terms-Cross-gain modulation, optical injection, wavelength conversion.

\section{INTRODUCTION}

A LL-OPTICAL wavelength converters are expected to become key components in future broadband networks. Wavelength conversion techniques include cross-gain modulation (XGM) or cross-phase modulation (XPM) in semiconductor optical amplifiers (SOA) [1]-[6], four-wave mixing (FWM) in passive waveguides [7], SOAs [8], or semiconductor lasers [9], gain-suppression mechanism in the semiconductor lasers such as DBR lasers [10], [11] and T-Gate lasers [13], laser-based wavelength conversion [14], [15], and difference frequency generation (DFG) [16].

Optical XGM in SOAs has been intensively studied in the past. However, there are relatively few papers on XGM in semiconductor lasers, especially small-signal modulation [18], [20], [28]. In this paper, we will concentrate on small-signal XGM in semiconductor lasers. An intensity-modulated input signal at a pump wavelength $\lambda_{2}$ is used to modulate the carrier density and consequently also the gain of a test laser due to gain saturation. In the test laser, a continuous wave (CW) beam at desired test wavelength $\lambda_{1}$ (called the test signal) is modulated by the gain variation. In this way, information is transferred from the pump wavelength to the test wavelength. The XGM response, which is obtained by pumping in the gain region of the quantum wells (QWs), is of great practical significance for wavelength con-

Manuscript received May 15, 2000; revised August 28, 2000. This work was supported by the Office of Naval Research under Grant N00014-96-1-0303 and Grant N00014-98-1-0604.

The authors are with the Department of Electrical and Computer Engineering, University of Illinois at Urbana-Champaign, Urbana, IL 61801 USA.

Publisher Item Identifier S 0018-9197(00)10562-7. version. The modulation response in this case will suffer virtually no adverse transport effects; hence, the response is practically intrinsic in nature, and shows a clear picture of the physical interactions taking place in the semiconductor laser. Our theoretical model also focuses on small-signal analysis, which is used to study the modulation bandwidth or wavelength conversion speed. If one is interested in bit-error rate, however, a large-signal approach is required [1], [4].

Several groups have measured the optical-absorption modulation response of a semiconductor laser for optical pumping within the QW region, where the pump photons create electron-hole pairs as they are absorbed [19], [23], [24]. The newly created carriers relax into the lower states of the QW, modulating the QW carrier density and the laser output. In this paper, optical pumping within the QWs is also investigated, but because the optical pump energy is chosen to coincide with the gain region of the test laser, certain physical interactions are distinctly different from the previous cross-absorption case [25].

When the optical pump wavelength is within the gain region of the test laser, the pump signal will be amplified through stimulated recombination of carriers rather than the creation of carriers through absorption. The amplification of the pump signal will have two major effects. First, the carrier lifetime will decrease because of stimulated recombination. Second, the test-laser intensity will decrease at a given bias when the pump signal is injected. The test-laser photon density and carrier lifetime significantly impact the modulation response of the laser. Moreover, there are effects which arise from cross-gain saturation due to the presence of more than one intense laser field which can also influence the modulation response.

In this study, XGM is studied at a pump [distributed feedback (DFB)] laser wavelength of $1542 \mathrm{~nm}$, which is in the gain region of the test laser. The effect of the pump intensity on the small-signal modulation response of the test signal is studied in detail. The paper is organized as follows. In Section II, the small-signal optical XGM theory is presented and compared with that of intrinsic intensity modulation response of a test laser under a direct microwave modulation without an external optical pump. In Section III, the experimental setup is described, and experimental results are shown and compared with the theory. The conclusion is presented in Section IV.

\section{Theory For An OpticAl Pump IN the Gain REgion With NONLINEAR CROSS-GAIN SATURATION}

Consider a pump laser (denoted by the subscript 2) with a photon density $S_{2}$ competing for the gain with a test laser (denoted by the subscript 1 ) with a photon density $S_{1}$. The rate 
equations for the carrier density $N\left(1 / \mathrm{cm}^{3}\right)$ and the photon density $S_{1}\left(1 / \mathrm{cm}^{3}\right)$ of the lasing mode (test signal) are

$$
\begin{aligned}
\frac{d N(t)}{d t}= & \frac{I}{q V}-\frac{N(t)}{\tau_{n}}-\frac{v_{g} G_{1} S_{1}(t)}{1+\epsilon_{11} S_{1}(t)+\epsilon_{12} S_{2}(t)} \\
& -\frac{v_{g} G_{2} S_{2}(t)}{1+\epsilon_{21} S_{1}(t)+\epsilon_{22} S_{2}(t)} \\
\frac{d S_{1}(t)}{d t}= & \frac{\Gamma v_{g} G_{1} S_{1}(t)}{1+\epsilon_{11} S_{1}(t)+\epsilon_{12} S_{2}(t)}-\frac{S_{1}(t)}{\tau_{p}}
\end{aligned}
$$

where

$I \quad$ test-laser current;

$V \quad$ volume of the active region;

$q \quad$ unit charge of the carrier;

$\tau_{n} \quad$ carrier lifetime;

$v_{g} \quad$ group velocity;

$\tau_{p} \quad$ photon lifetime;

$\Gamma \quad$ optical confinement factor;

$G_{1,2}$ gain at the test and pump laser wavelength, respectively.

In order to take into account the effects of nonlinear gain suppression with cross-gain-saturation, we include $\epsilon_{11}$ and $\epsilon_{22}$, which are the self-nonlinear gain saturation coefficients, and $\epsilon_{12}$ and $\epsilon_{21}$, which are the cross-nonlinear gain saturation coefficients. The cross-saturation properties of the gain due to pump-test-laser interactions describe how the pump and test signals interact with each other in the active region. The gain suppression at a wavelength $\lambda_{1}$ will be due to the presence of both the test and pump photon densities, although not necessarily to the same degree. The spontaneous emission term has been neglected because the test laser is above threshold.

\section{A. Steady-State Solution}

In the steady state, the time-varying terms are set to zero in the rate equations (1) and (2). The equation for the photon density is used to define the steady-state gain-loss relation

$$
\frac{1}{\tau_{p}}=\frac{\Gamma v_{g} G_{1}}{1+\epsilon_{11} S_{1}+\epsilon_{12} S_{2}} .
$$

For simplicity in notation, capital letters $S_{1}$ and $S_{2}$ stand for steady-state values. The equation for the carrier density can also be used to solve for the light-current $(L-I)$ characteristics of the test laser, after setting the time-varying terms to zero

$$
\begin{aligned}
I- & \frac{q V N_{0}}{\tau_{n}}-q V v_{g} \frac{G_{2} S_{2}}{1+\epsilon_{21} S_{1}+\epsilon_{22} S_{2}} \\
& =q V v_{g} \frac{G_{1} S_{1}}{1+\epsilon_{11} S_{1}+\epsilon_{12} S_{2}}
\end{aligned}
$$

where $I_{t h}=q V N_{0} / \tau_{n}$ is the original threshold current without an external pump. With cross saturation, the $L-I$ relationship may not behave as a simple, linear function. For a given testlaser current $I$, the photon density of the test-laser $S_{1}$ will be less than what it would be if $S_{2}$ were not present, since the pump $S_{2}$ competes for carriers, causing both a shift in threshold for the test laser and a change in the slope of its $L-I$ curve.

\section{B. Small-Signal Solution}

In this section, the changes in the lasing mode photon densities and carrier density due to the pump signal variation are assumed to be much smaller than the steady-state value of the photon and carrier densities. To solve for the small-signal modulation response, the expressions for carrier and photon densities are

$$
\begin{aligned}
& N(t)=N_{0}+n(\omega) e^{j \omega t} \\
& S_{1}(t)=S_{1}+s_{1}(\omega) e^{j \omega t} \\
& S_{2}(t)=S_{2}+s_{2}(\omega) e^{j \omega t}
\end{aligned}
$$

and by linearizing the gain function

$$
G_{1,2}(N)=G_{1,2}\left(N_{0}\right)+g_{1,2}^{\prime}\left(N-N_{0}\right)
$$

where $g_{1,2}^{\prime}$ is the differential gain at wavelength $\lambda_{1}$ or $\lambda_{2}$. For the small-signal analysis, the quantity $N-N_{0}$ will equal the small-signal change in carrier density, denoted by $n$.

Taylor's series expansion is used to simplify the small-signal form of the rate equations. Note that the source of modulation is the pump photon density. Terms containing products of steadystate and small-signal components are linearized, and only firstorder terms are retained. The small-signal rate equations can be expressed as follows:

$$
\begin{aligned}
j \omega n= & -\frac{n}{\tau_{n}} \\
& -v_{g}\left(\frac{g_{1}^{\prime} n S_{1}+G_{1} s_{1}}{1+\epsilon_{11} S_{1}+\epsilon_{12} S_{2}}-\frac{G_{1} S_{1}\left(\epsilon_{11} s_{1}+\epsilon_{12} s_{2}\right)}{\left(1+\epsilon_{11} S_{1}+\epsilon_{12} S_{2}\right)^{2}}\right) \\
& -v_{g}\left(\frac{g_{2}^{\prime} n S_{2}+G_{2} s_{2}}{1+\epsilon_{21} S_{1}+\epsilon_{22} S_{2}}-\frac{G_{2} S_{2}\left(\epsilon_{21} s_{1}+\epsilon_{22} s_{2}\right)}{\left(1+\epsilon_{21} S_{1}+\epsilon_{22} S_{2}\right)^{2}}\right) \\
j \omega s_{1}= & \Gamma v_{g}\left(\frac{g_{1}^{\prime} n S_{1}+G_{1} s_{1}}{1+\epsilon_{11} S_{1}+\epsilon_{12} S_{2}}\right. \\
& \left.-\frac{G_{1} S_{1}\left(\epsilon_{11} s_{1}+\epsilon_{12} s_{2}\right)}{\left(1+\epsilon_{11} S_{1}+\epsilon_{12} S_{2}\right)^{2}}\right)-\frac{s_{1}}{\tau_{p}}
\end{aligned}
$$

After eliminating the carrier density $\mathrm{n}$ and solving for $s_{1} / s_{2}$, the response is obtained

$$
M(\omega)=\frac{s_{1}(\omega)}{s_{2}(\omega)}=\frac{N(\omega)}{-\omega^{2}-j \omega \gamma+\omega_{r}^{2}}
$$

where the numerator $N(\omega)$ is

$$
\begin{aligned}
N(\omega)= & \frac{\epsilon_{12} S_{1}}{\tau_{p}\left(1+\epsilon_{11} S_{1}+\epsilon_{12} S_{2}\right)} \\
& \cdot\left(j \omega-\frac{1}{\tau_{n}^{\prime}}-X\left(1+\epsilon_{21} S_{1}\right) \frac{G_{2}}{G_{1}} \frac{v_{g} g_{1}^{\prime}}{\epsilon_{12}}\right)
\end{aligned}
$$

in which the effective carrier lifetime $\tau_{n}{ }^{\prime}$ due to stimulated recombination by the pump $S_{2}$ is defined as

$$
\frac{1}{\tau_{n}^{\prime}}=\frac{1}{\tau_{n}}+\frac{v_{g} g_{2}^{\prime} S_{2}}{1+\epsilon_{21} S_{1}+\epsilon_{22} S_{2}}
$$


TABLE I

COMPARISON OF INTENSITY MODULATION RESPONSES: INTRINSIC AND XGM

\begin{tabular}{|c|c|}
\hline \multicolumn{2}{|c|}{ Optical (in-well) Pumping } \\
\hline Intrinsic & Cross-gain modulation \\
\hline$\frac{M(\omega)}{M(0)}=\frac{\omega_{r}^{2}}{\omega_{r}^{2}-\omega^{2}+j \omega \gamma}$ & $\frac{M(\omega)}{M(0)}=\frac{\omega_{r}^{2}}{\omega_{r}^{2}-\omega^{2}+j \omega \gamma}$ \\
\hline$\gamma=\frac{1}{\tau_{n}}+\frac{v_{g} g_{1}^{\prime} S_{1}}{\tau_{p}\left(1+\epsilon S_{1}\right)}\left(\tau_{p}+\frac{\epsilon}{v_{g} g_{1}^{\prime}}\right)$ & $\gamma=\frac{1}{\tau_{n}^{\prime}}+\frac{v_{g} g_{1}^{\prime} S_{1}}{\tau_{p}\left(1+\epsilon_{11} S_{1}+\epsilon_{12} S_{2}\right)}\left(\tau_{p}+\frac{\epsilon_{11}}{v_{g} g_{1}^{\prime}}\right)$ \\
\hline$\omega_{r}^{2}=\frac{v_{g} g_{1}^{\prime} S_{1}}{\tau_{p}\left(1+\epsilon S_{1}\right)}\left(1+\frac{\epsilon}{\tau_{n} v_{g} g_{1}^{\prime}}\right)$ & $\omega_{r}^{2}=\frac{v_{g} g_{1}^{\prime} S_{1}}{\tau_{p}\left(1+\epsilon_{11} S_{1}+\epsilon_{12} S_{2}\right)}\left(1+\frac{\epsilon_{12}}{\tau_{n}^{\prime} v_{g} g_{1}^{\prime}}-X \frac{G_{1}}{G_{2}} \epsilon_{21} S_{2}\right)$ \\
\hline$\gamma=K f_{r}^{2}+\frac{1}{\tau_{n}}$ & $\gamma=K f_{r}^{2}+\frac{1}{\tau_{n}^{\prime}}$ \\
\hline$K=4 \pi^{2} \frac{\tau_{p}+\frac{\epsilon}{v_{g} g_{1}^{T}}}{1+\frac{\epsilon}{\tau_{n} v_{g} g_{1}^{T}}}$ & $K=4 \pi^{2} \frac{\tau_{p}+\frac{\epsilon_{11}}{v_{g} g_{1}^{\prime}}}{1+\frac{\epsilon_{12}}{\tau_{n}^{\prime} v_{g} g_{1}^{\prime}}-X \frac{G_{1}}{G_{2}} \epsilon_{21} S_{2}}$ \\
\hline & $\frac{1}{\tau_{n}^{\prime}}=\frac{1}{\tau_{n}}+\frac{v_{g} g_{2}^{\prime} S_{2}}{1+\epsilon_{21} S_{1}+\epsilon_{22} S_{2}}$ \\
\hline & $X=\frac{1+\epsilon_{12} S_{1}+\epsilon_{12} S_{2}}{\left(1+\epsilon_{21} S_{1}+\epsilon_{22} S_{2}\right)^{2}}$ \\
\hline
\end{tabular}

and the cross-gain-saturation term $X$ is

$$
X=\frac{1+\epsilon_{11} S_{1}+\epsilon_{12} S_{2}}{\left(1+\epsilon_{21} S_{1}+\epsilon_{22} S_{2}\right)^{2}} .
$$

Now the damping factor can be defined, after simplification, as

$$
\gamma=\frac{1}{\tau_{n}^{\prime}}+\frac{v_{g} g_{1}^{\prime} S_{1}}{\tau_{p}\left(1+\epsilon_{11} S_{1}+\epsilon_{12} S_{2}\right)}\left(\tau_{p}+\frac{\epsilon_{11}}{v_{g} g_{1}^{\prime}}\right)
$$

and the resonant frequency squared may be written as

$$
\omega_{r}^{2}=\frac{S_{1} v_{g} g_{1}^{\prime}}{\tau_{p}\left(1+\epsilon_{11} S_{1}+\epsilon_{12} S_{2}\right)}\left(1+\frac{\epsilon_{11}}{\tau_{n}^{\prime} v_{g} g_{1}^{\prime}}-X \frac{G_{1}}{G_{2}} \epsilon_{21} S_{2}\right)
$$

or replacing $1 / \tau_{n}^{\prime}$ by $1 / \tau_{n}$ using (14)

$$
\omega_{r}^{2}=\frac{S_{1} v_{g} g_{1}^{\prime}}{\tau_{p}\left(1+\epsilon_{11} S_{1}+\epsilon_{12} S_{2}\right)}\left(1+\frac{\epsilon_{11}}{\tau_{n} v_{g} g_{1}^{\prime}}+Y S_{2}\right)
$$

where

$$
Y=\frac{\epsilon_{11}}{1+\epsilon_{21} S_{1}+\epsilon_{22} S_{2}}-\frac{\epsilon_{21} \frac{G_{1}}{G_{2}}\left(1+\epsilon_{11} S_{1}+\epsilon_{12} S_{2}\right)}{\left(1+\epsilon_{21} S_{1}+\epsilon_{22} S_{2}\right)^{2}} .
$$

The expression for the damping factor remains almost the same, except for the reduced carrier lifetime. The relaxation frequency $\left(\omega_{r}=2 \pi f_{r}\right)$, however, depends on pump laser photon density $S_{2}$. The overall response is simply the "intrinsic" form of the response in the denominator, but with different values defining the relaxation frequency $\omega_{r}$ and the damping factor $\gamma$. Equations (14), (16), and (17) indicate new analytical results on the effective inverse carrier lifetime $\left(1 / \tau_{n}^{\prime}\right), \gamma$, and $\omega_{r}^{2}$, respectively. The numerator $N(\omega)$ remains almost constant within the frequency range of interest. As a final step, the overall response is normalized, and the magnitude is written as

$$
\left|\frac{M(\omega)}{M(0)}\right|^{2} \simeq \frac{\omega_{r}^{2}}{\left(\omega_{r}^{2}-\omega^{2}\right)^{2}+\omega^{2} \gamma^{2}} .
$$

The equations are summarized in Table I. The expressions for the conventional intrinsic small-signal modulation response are also listed in Table I for comparison. It should be noted that the two sets of modulation responses are identical when the photon density $S_{2}$ approaches zero. Therefore, the expressions for the small-signal optical gain modulation response are actually the intrinsic modulation response of the semiconductor laser and are useful in studying the physics of XGM. 
TABLE II

STRUCTURE OF THE TEST LASER

\begin{tabular}{lllll}
\hline Well: & Number of wells & 5 & SCH width: & $1000 \AA$ \\
& Material & InGaAlAs & Stripe width: & $1.5 \mu m$ \\
& Strain & $-0.78 \%$ comp. & Cavity length: & $545 \mu m$ \\
& Width & $54 \AA$ & & \\
& PL wavelength & $1.56 \mu m$ & & \\
\hline Barrier: & Material & InGaAlAs & & \\
& Strain & lattice matched & \\
& Width & $57 \AA$ & & \\
& PL wavelength & $1.21 \mu m$ & & \\
\hline
\end{tabular}

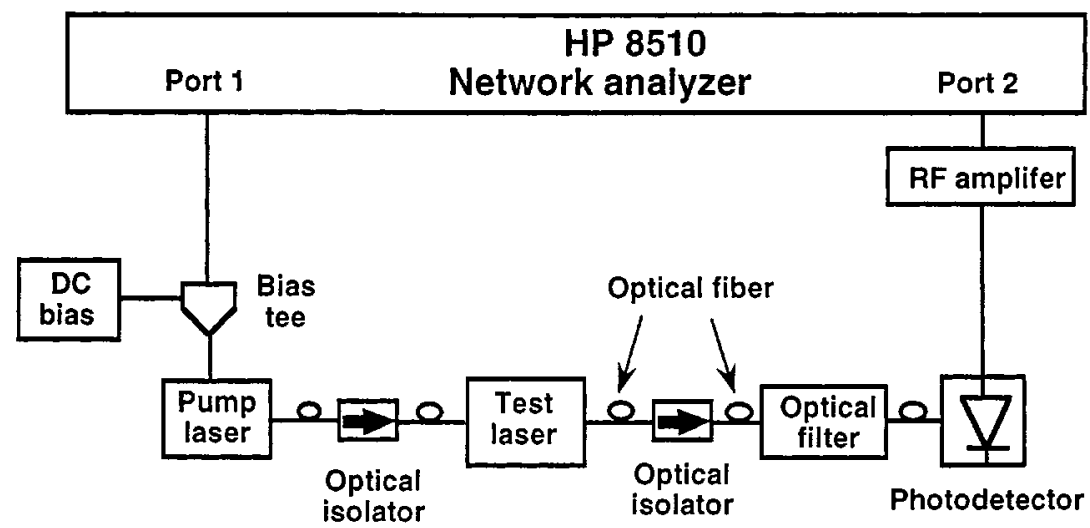

Fig. 1. Experimental setup for the optical XGM measurement. The pump laser is a DFB laser emitting at a single-wavelength within the gain spectrum of the test laser. The test laser is an InAlGaAs QW laser, which can be directly modulated by current or optically modulated by the pump laser.

\section{EXPERIMENTAL SETUP AND RESULTS}

\section{A. Experimental Setup}

Optical-gain modulation experiments are performed on a multiple-QW InGaAlAs Fabry-Perot (FP) laser, which is used as the test laser. The composition of the undoped active region is described in Table II. The barrier photoluminescence wavelength is $1.21 \mu \mathrm{m}$, and the photoluminescence wavelength peak of the active region is $1.56 \mu \mathrm{m}$. We select a pump laser wavelength around $1.542 \mu \mathrm{m}$, which is in the gain region of the test laser [27].

A schematic diagram of the gain-modulation response experiment is shown in Fig. 1. The pump laser is a $1.542-\mu \mathrm{m}$ DFB) laser with a 3-dB bandwidth of $9 \mathrm{GHz}$. The pump laser is modulated electrically, and its optical output is injected into the test laser. The XGM is realized in the test-laser cavity. The overall modulation response of the test laser has been calibrated with respect to the modulation response of the pump laser. The modulated pump and test signals are injected from the test laser into a fiber using a lensed fiber-optical interface. The test-laser output is filtered through and converted into a microwave signal using a $29-\mathrm{GHz}$ high-speed photodetector. The microwave signal is then amplified using a 18-dB-gain microwave amplifier, and the small-signal responses $|M(\omega)|^{2}$ are measured by an HP8510 network analyzer. The normalized modulation response $|M(\omega) / M(0)|^{2}$ is obtained according to the low-frequency value $|M(0)|^{2}$, which is referred to as the signal level. The optical XGM experiments are performed in a manner similar to the optical pump experiments of pumping in the absorption region in [24]. However, there are a few important differences in procedure which must be followed. First of all, the light coupled from the output of the test laser includes both the pump signal and the test signal. Therefore, the output must be filtered to remove the pump light before the light enters the high-speed detector. In the experiments, a tunable fiber-optic bandpass filter is used to capture only the light from the test laser, and reject all of the light at the pump laser wavelength. Secondly, the dc portion of the pump light causes a shift in the test-laser threshold, decreases the test-laser intensity at a given bias, and experiences amplification as it passes through the laser. It is essential to monitor both the pump and test-laser intensities at each value of bias current, and to measure the test-laser intensity with the pump on and off. Finally, the pump laser should be chosen to have a wavelength sufficiently detuned from the eigen-frequency of the test-laser cavity to avoid injection locking [29].

\section{B. Dc Analysis}

Because gain saturation is an intensity-dependent phenomenon, it is important to understand the effects of dc bias upon optical injection. In addition to explaining the experimental data using our model, we would like to discuss major possible phenomena of optical gain modulation, which is not only limited 
in the behavior of our particular test laser. From our theory, the new threshold and the slope of the $L-I$ curve including these effects are

$$
\begin{aligned}
I_{\text {th-new }} & =\frac{q V N_{0}}{\tau_{n}}+q V v_{g}\left(\frac{G_{2} S_{2}}{1+\epsilon_{21} S_{1}+\epsilon_{22} S_{2}}\right) \\
\text { Slope } & =q V v_{g}\left(\frac{G_{1}}{1+\epsilon_{11} S_{1}+\epsilon_{12} S_{2}}\right) .
\end{aligned}
$$

When the cross-saturation coefficients are large, the "slope" will decrease rapidly with a larger pumping, and the increase in threshold will be slow. If the saturation effect is negligible, we will not observe the slope change of the $L-I$ curve. The output power of the probe laser degrades only because of the rapidly increasing threshold.

Fig. 2 shows the $L-I$ curves of the test laser at different pump powers. The data were taken while measuring the XGM responses. The symbols are experimental data, and the lines show their trends. In this case, the pump causes a shift in the threshold and significantly alters the slope of the $L-I$ curve, which indicates large cross-gain-saturation effects.

\section{Small-Signal XGM}

The small-signal amplitude-modulation response of the test signal is measured when an intensity-modulated pump signal is injected into the test laser. Fig. 3(a) shows the normalized modulation response of the test laser under a constant pump laser injection $(0.8 \mathrm{~mW})$ at different test-laser biases $(15,25$, and 40 $\mathrm{mA})$. The power of the pump laser is measured before it is injected into the test laser. The modulation responses clearly show relaxation frequencies at 2, 5.2, and $6.5 \mathrm{GHz}$, respectively. For comparison, the normalized small-signal electrical modulation of the test laser is also presented in Fig. 3(b). The relaxation frequency peaks are at 2.1, 4.8, and $6.2 \mathrm{GHz}$ at biases of 15 , 25 , and $40 \mathrm{~mA}$, respectively. Optical modulation directly modulates the carrier density in the same manner as current modulation (electrical modulation), but removes carrier transport effects and the circuit parasitics, which contribute to an additional low-frequency roll-off in the modulation response [24]. At low current bias, even the relaxation frequencies of electrical and optical modulation are close, and the optical gain modulation still shows a higher relaxation peak. In general, the optical gain modulation can improve modulation bandwidth compared to the electrical modulation.

By fitting the frequency response function in (20) to the experimental data, the damping factor $\gamma$ and the relaxation frequency $\omega_{r}$ at different test-laser biases and pumping powers are obtained as shown in Fig. 4. At a constant relaxation frequency, the damping factor increases with increasing pump signal, and the modulation response is flattened. The slope of the linear fit of the damping factor versus relaxation frequency squared is the $K$ factor

$$
\gamma=K f_{r}^{2}+\frac{1}{\tau_{n}^{\prime}}
$$

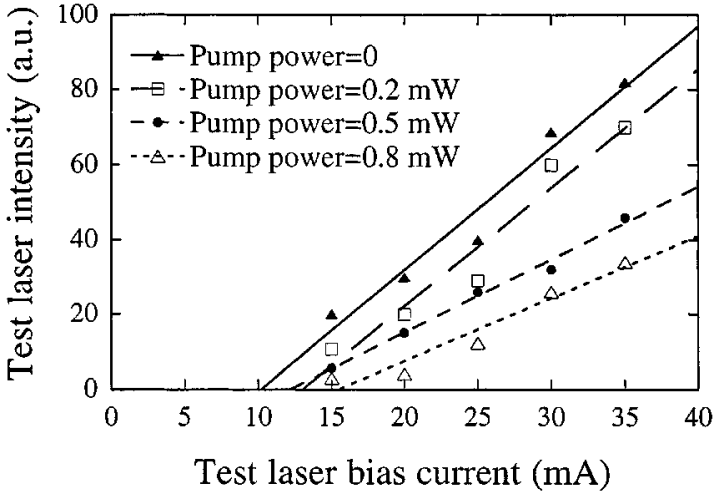

Fig. 2. Light output versus injection current of the test laser for external pump signal injection of $0,0.2,0.5$, and $0.8 \mathrm{~mW}$. The symbols are experimental data, and the lines show the trends.
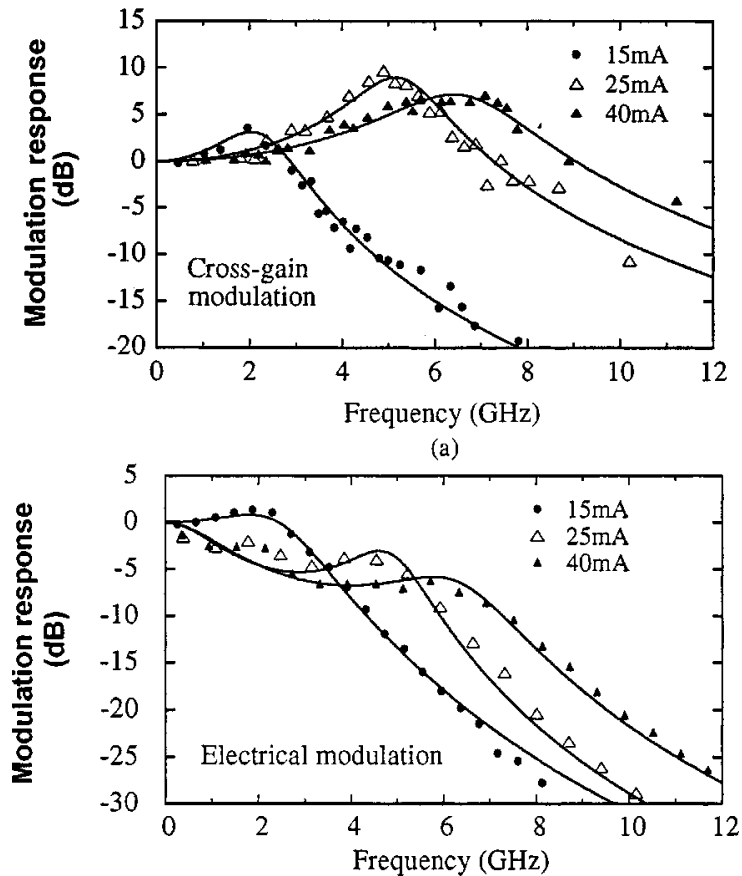

(b)

Fig. 3. (a) The XGM response of the test laser at bias currents of 15,25 , and 40 $\mathrm{mA}$ with a pump laser power of $0.8 \mathrm{~mW}$ using the setup in Fig. 1. The symbols represent the experimental data. The solid curves are theoretical fitting. (b) The electrical small-signal intensity modulation response of the test laser (symbols) is plotted at bias currents of 15,25 , and $40 \mathrm{~mA}$. No external pump laser light is injected. The solid curves are theoretical fitting.

$$
K=4 \pi^{2} \frac{\tau_{p}+\frac{\epsilon_{11}}{v_{g} g_{1}^{\prime}}}{1+\frac{\epsilon_{11}}{\tau_{n}^{\prime} v_{g} g_{1}^{\prime}}-X \frac{G_{1}}{G_{2}} \epsilon_{21} S_{2}} .
$$

Fig. 5(a) shows that the $K$ factor increases with increasing pump power, as predicted by (24), but the change is very small. In Fig. 4, the vertical axis-intercept of the linear fit can be used to calculate the effective carrier lifetime $\tau_{n}^{\prime}$. The effective carrier lifetime versus pumping power is shown in Fig. 5(b). We can see the reduction of the effective carrier lifetime when the pump light is injected, as expected from theory (14), because the amplification of the pump signal in the test laser increases the stimulated recombination rate. The solid lines in Fig. 5 are 


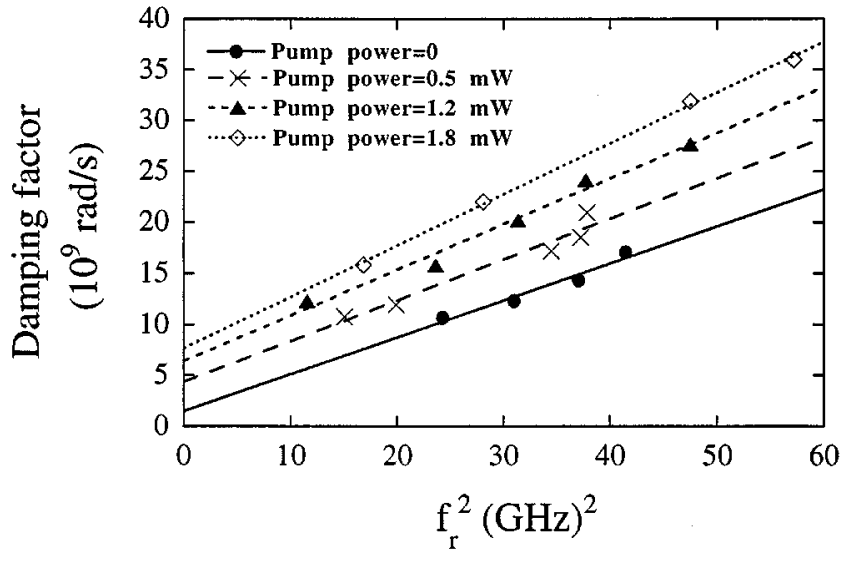

Fig. 4. Damping factor versus the relaxation frequency squared at different pump power injection. The symbols are experimental data and the lines are the linear fitting.

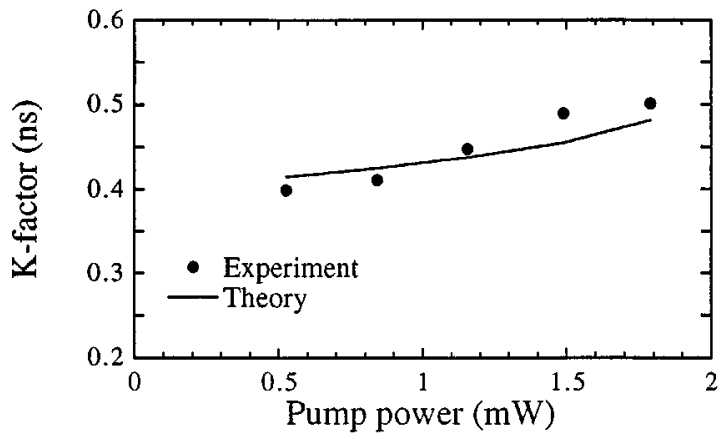

(a)

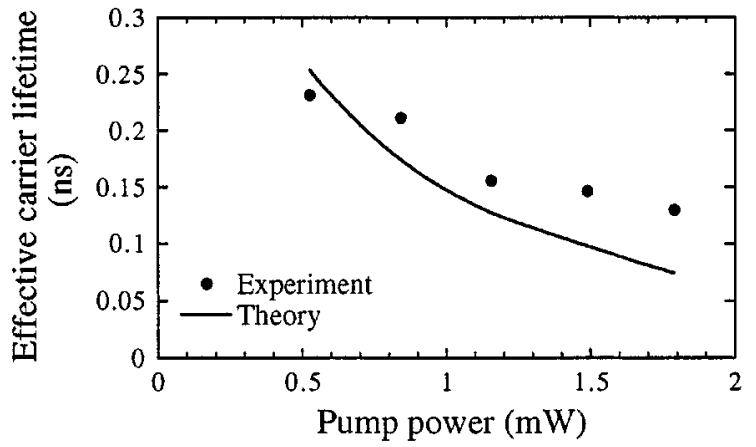

(b)

Fig. 5. (a) The $K$ factor and (b) the effective carrier lifetime versus pump injection power. The solid lines are theoretical calculations.

the theoretical calculations, which agree very well with our experimental data. In our calculation, some of the parameters such as the effective index of refraction, intrinsic loss, and the initial value of the differential gain are obtained from previous independent measurements [27]. The effective index of refraction of the test laser is $n_{g}=3.3$, which can be used to calculate the group velocity $v_{g}=c / n_{g}=9.1 \times 10^{9} \mathrm{~cm} / \mathrm{s}$, the facet reflection coefficient $R=\left(n_{g}-1\right)^{2} /\left(n_{g}+1\right)^{2}=0.29$, mirror loss $\alpha_{m}=-(1 / L) \ln R=23 \mathrm{~cm}^{-1}$, and photon lifetime $\tau_{p}=1 /\left[v_{g}\left(\alpha_{i}+\alpha_{m}\right)\right]=2.1$ ps. The final value of the differential gain, self-, and cross-gain saturation coefficients are extracted by fitting the $K$-factor and the effective carrier lifetime. To simplify the calculation, we assume $g_{1}^{\prime}=g_{2}^{\prime}$ because the wavelength of the pump signal and the test signal are very
TABLE III

THE TEST LASER PARAMETERS

\begin{tabular}{llll}
\hline Parameter & Symbols & Unit & Value \\
\hline Test laser wavelength & $\lambda_{1}$ & $\mathrm{~nm}$ & 1552 \\
Pump laser wavelength & $\lambda_{2}$ & $\mathrm{~nm}$ & 1542 \\
Test laser gain bandwidth at $12 \mathrm{~mA}$ & & $\mathrm{~nm}$ & $1490-1600$ \\
Cavity length & $\mathrm{L}$ & $\mu$ & 545 \\
Active volume & $V$ & $\mathrm{~cm}^{3}$ & $3.2 \times 10^{-10}$ \\
Effective index of refraction & $n_{g}$ & & 3.3 \\
Group velocity & $v_{g}$ & $\mathrm{~cm} / \mathrm{s}$ & $9.1 \times 10^{9}$ \\
Mirror loss & $\alpha_{m}$ & $\mathrm{~cm}^{-1}$ & 23 \\
Intrinsic loss & $\alpha_{i}$ & $\mathrm{~cm}^{-1}$ & 30 \\
Photon lifetime & $\tau_{p}$ & $\mathrm{ps}$ & 2.1 \\
Carrier lifetime & $\tau_{n}$ & $\mathrm{~ns}$ & 0.5 \\
Differential gain & $g_{1}^{\prime}=g_{1}^{\prime}$ & $\mathrm{cm}^{2}$ & $3 \times 10^{-17}$ \\
Nonlinear self-gain saturation coefficient & $\epsilon_{11}=\epsilon_{22}$ & $\mathrm{~cm}^{3}$ & $2.2 \times 10^{-17}$ \\
Nonlinear cross-gain saturation coefficient & $\epsilon_{12}=\epsilon_{21}$ & $\mathrm{~cm}^{3}$ & $1.5 \times 10^{-17}$ \\
\hline
\end{tabular}

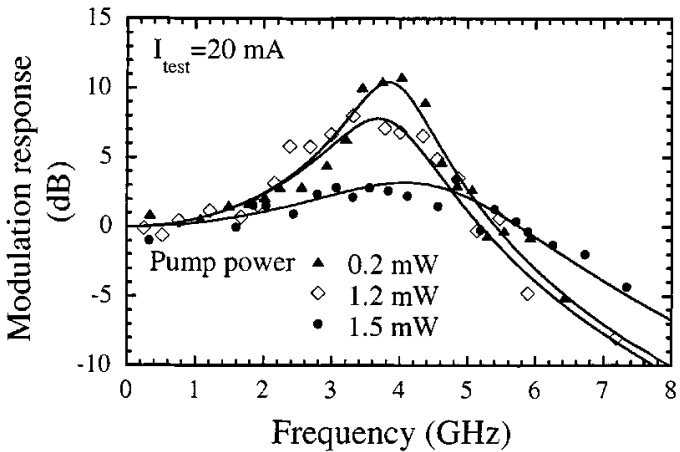

(a)

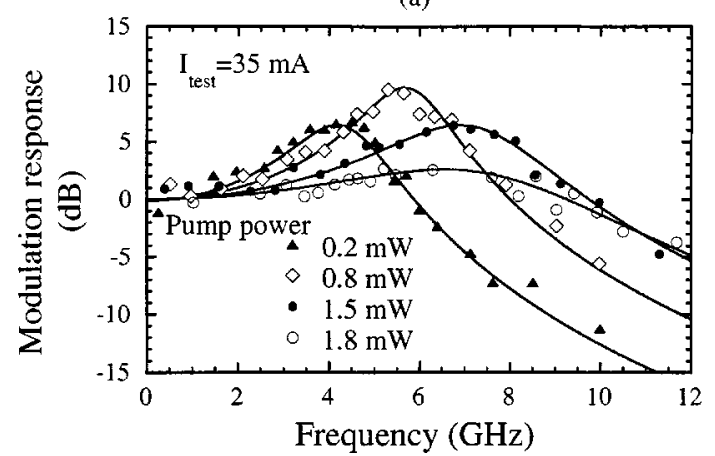

(b)

Fig. 6. (a) XGM response of the test wavelength with a fixed test-laser bias of $20 \mathrm{~mA}$ and pump powers of $0.2,1.2$, and $1.5 \mathrm{~mW}$. (b) XGM response of the test wavelength with a fixed test-laser bias of $35 \mathrm{~mA}$ and pump powers of 0.2 , $0.8,1.5$, and $1.8 \mathrm{~mW}$. The symbols are experimental data, and solid curves are theoretical fitting.

close. The test-laser parameters used for the theoretical calculation are listed in Table III.

The effect of pump power on the modulation responses was studied, focusing on the effects of the pump signal on the relaxation frequency and the damping factor. Fig. 6(a) shows the normalized response of the test laser with fixed test laser bias (20 $\mathrm{mA}$ ) and pump powers of $0.2,1.2$, and $1.5 \mathrm{~mW}$. Fig. 6(b) shows the normalized response of the test laser with a higher fixed test-laser bias (35 $\mathrm{mA})$ and pump powers of $0.2,0.8,1.5$, and $1.8 \mathrm{~mW}$. At low test-laser bias $(20 \mathrm{~mA})$, the relaxation frequency 
is almost unchanged with different pump injection. This is because the dc injection current is low and the pump signal consumes most of the available carriers. An increase of pump power saturates the cavity gain, reduces the test-laser photon density, and flattens the relaxation peak of modulation responses. Further increasing pumping power can switch the test laser below threshold, and no modulation response can be observed, while at high test-laser bias (35 mA), more carriers are available, and the relaxation frequency increases with increasing pump signal. The modulation response is improved with increasing pumping power, while the improvement of the modulation bandwidth has its limit. If we further increase the pump signal to $1.8 \mathrm{~mW}$, the modulation responses show large damping at the test wavelength, and the modulation bandwidth improves very little. At the same time, we also observe a decrease in the signal level $|M(0)|^{2}$. The damping factor at low injection is much smaller than that at high injection, as shown in Fig. 4. Therefore, at higher pumping, the test-laser modulation response is dominated by the high damping from cross-gain-saturation. When the test-laser dc bias is increased further, the test-laser signal will experience more saturation, and the test signal level will decrease further. Although the relaxation frequency will increase as shown in Fig. 6(b), the overall modulation bandwidth will not be improved much, and will reach its limit for the optical gain modulation because of the huge damping. In general, the increase of the damping factor due to cross-gain saturation limits the improvement of the bandwidth.

Fig. 7 shows the relaxation frequency squared versus testlaser bias at different pump powers. The lines show the linear trends of the squared relaxation frequency versus test-laser bias. At low test-laser bias (15 mA) and high pump injection (above $1.2 \mathrm{~mW}$ ), the pump drives the test laser into below-threshold operation, and no modulation signal can be measured. In Fig. 7, the interception of the linear fit with the horizontal axis is around the new threshold of the test laser. The data also show the trend of threshold increment with higher injection. The variation of the relaxation frequency with pumping signal can be explained by our model. As shown in (18) and (19), the Y factor depends mostly on the saturation coefficients. If the self-gain-saturation coefficients $\epsilon_{\ddot{i}}$ are bigger than the cross-gain-saturation coefficients $\epsilon_{i j}$, we can show that the $\mathrm{Y}$ factor is positive. There are several effects of pump signal on the test signal. First, the pump signal shifts the threshold of the test laser and also reduces the photon density of the test wavelength $S_{1}$, which will decrease the relaxation frequency. Second, because of the selfand cross-gain-saturation, when the $\mathrm{Y}$ factor is positive, the relaxation frequency can also increase with higher injection. The overall variation of the relaxation frequency depends on which effects are dominant. For our experiment, at low test-laser bias, since $S_{1}$ is small, the percentage reduction of test signal photon density is large, and the first effect is compatible with the second effect. We observe a small decrease of the relaxation frequency with increasing pump power. Actually, the variation of the relaxation frequency is very small because of the small $S_{1}$ value and the cancellation of the two effects. On the other hand, at large test-laser bias, the second effect is more important than the first for our test laser. The percentage variation of photon density $S_{1}$ with different pump power is small, and a large $S_{1}$

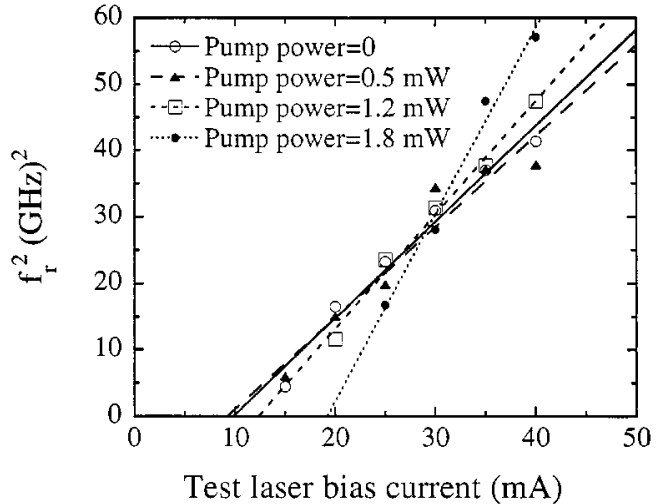

Fig. 7. The relaxation frequency squared versus the test-laser bias. The symbols are results for experimental data, and the lines are linear fitting.

value amplifies the effect of $S_{2}$ variation, which results in a large relaxation frequency difference. Fig. 7 indicates small changes of relaxation frequency at low test-laser bias, and a large relaxation frequency variation at high bias.

\section{CONCLUSION}

We have theoretically and experimentally evaluated the small-signal intensity modulation of the QW test laser driven by the injection of modulated pump signal. Rate equations for optical pumping within the gain region, including the effects of pump-induced stimulated recombination and cross-gain saturation, which corresponds to wavelength conversion by XGM, have been presented. The model predictions, such as a shortened carrier lifetime and a shift in the laser $L-I$ threshold, are confirmed by the experiments. The model also well explains the variation of the relaxation frequency with different pump power. The test-laser photon density and carrier lifetime both influence significantly the modulation responses and show major features in XGM. Our experiments and theory also show that there are subtle effects from cross-gain-saturation due to the presence of two laser fields affecting the optical gain modulation response. Comparison between electrical modulation and optical XGM of the test laser is also presented, which shows improvement of modulation bandwidth by optical XGM.

\section{ACKNOWLEDGMENT}

The authors would like to thank Dr. T. Tanbun-Ek for providing the QW lasers for this study and Dr. T. C. Wu for providing expert advice on the optical modulation experiments.

\section{REFERENCES}

[1] S. J. B. Yoo, "Wavelength conversion technologies for WDM network application,” J. Lightwave Technol., vol. 14, pp. 955-966, 1996.

[2] T. Durhuus, B. Mikkelsen, C. Joergense, S. L. Danielsen, and K. E. Stubkjaer, "All-optical wavelength conversion by semiconductor optical amplifier,” J. Lightwave Technol., vol. 14, pp. 942-954, 1996.

[3] M. Asghari, I. H. White, and R. V. Penty, "Wavelength conversion using semiconductor optical amplifiers," J. Lightwave Technol., vol. 15, pp. 1181-1190, 1997.

[4] K. Obermann, S. Kindt, D. Breuer, and K. Petermann, "Performance analysis of wavelength conversion based on cross-gain modulation in the semiconductor-optical amplifiers," J. Lightwave Technol., vol. 16, pp. 78-85, 1998 . 
[5] J. M. Wiesenfeld, B. Glance, J. S. Perino, and A. H. Gnauck, "Wavelength conversion at $10 \mathrm{~Gb} / \mathrm{s}$ using a semiconductor optical amplifier," IEEE Photon. Technol. Lett., vol. 5, pp. 1300-1306, 1993.

[6] S. Dubovitsky, A. Mathur, W. H. Steier, and P. D. Dapkus, "Gain saturation properties of a polarization insensitive semiconductor amplifier implemented with tensile and compressive strain quantum wells," IEEE Photon. Technol. Lett., vol. 6, pp. 176-178, 1994.

[7] K. Inoue, "Tunable and selective wavelength conversion using fiber four-wave mixing with two pump lights," IEEE Photon. Technol. Lett., vol. 6, pp. 1451-1453, 1994.

[8] R. Schnabel, U. Hilbk, T. Hermes, P. MeiBner, C. Helmolt, K. Magari, F. Raub, W. Pieper, F. J. Westphal, R. Ludwig, L. Kuller, and H. G. Weber, "Polarization insensitive frequency conversion of a 10-channel OFDM signal using four-wave-mixing in semiconductor laser amplifier," IEEE Photon. Technol. Lett., vol. 6, pp. 56-58, 1994.

[9] J. Minch, C. S. Chang, and S. L. Chuang, "Four-wave-mixing in a distributed-feedback laser," Appl. Phys. Lett., vol. 70, pp. 1360-1362, 1997.

[10] T. Durhuus, R. J. S. Pedersen, B. Mikkelsen, K. E. Stubkjaer, M. Oberg, and S. Nilsson, "Optical wavelength conversion over $18 \mathrm{~nm}$ at $2.5 \mathrm{~Gb} / \mathrm{s}$ by DBR-laser," IEEE Photon. Technol. Lett., vol. 5, pp. 86-88, 1993.

[11] H. Yasaka, K. Takahata, K. Kasaya, and K. Oe, "High-speed signal wavelength conversion using a unidirectional-output wavelength conversion device with asymmetric-k DBR structure," IEEE J. Quantum Electron., vol. 31, pp. 82-91, 1995.

[12] H. Kawaguchi, K. Magari, H. Yasaka, M. Fukuda, and K. Oe, "Tunable optical-wavelength conversion using an optically triggerable multielectrode distributed feedback laser diode," IEEE J. Quantum Electron., vol. 24, pp. 2153-2159, 1988.

[13] C. C. Lu, S. Jiang, P. S. Yeh, P. J. S. Heim, C. E. C. Wood, and M. Dagenais, "Wavelength conversion using a T-Gate laser," IEEE Photon. Technol. Lett., vol. 8, pp. 52-54, Jan. 1996.

[14] H. Sanjoh, H. Mawatari, H. Ishii, H. Yasaka, Y. Yoshikuni, and K. Oe, "Wavelength chirping in wavelength conversion of $10 \mathrm{~Gb} / \mathrm{s}$ signal with semiconductor laser converter," IEEE Photon. Technol. Lett., vol. 8, pp. 296-298, 1996

[15] M. F. C. Stephens, D. Nesset, K. A. Williams, A. E. Kelly, R. V. Penty, I. H. White, and M. J. Fice, "Wavelength conversion at $40 \mathrm{Gbit} / \mathrm{s}$ via cross-gain modulation in distributed feedback laser integrated with semiconductor optical amplifier," Electron. Lett., vol. 35, pp. 1762-1764, 1999.

[16] C. Q. Xu, H. Okayama, and M. Kawahara, " $1.5 \mu \mathrm{m}$ band efficient broadband wavelength conversion by difference frequency generation in a periodically domain-inverted $\mathrm{LiNbO}_{3}$ channel wavelength," Appl. Phys. Lett., vol. 63, pp. 3559-3561, 1993.

[17] M. P. Kesler and E. P. Ippen, "Subpicosecond gain dynamics in GaAlAs laser diodes," Appl. Phys. Lett., vol. 51, pp. 1765-1767, 1987.

[18] C. H. Lange and C. B. Su, "Theory and experiment of the parasitic-free frequency response measurement technique using facet-pumped optical modulation in semiconductor diode lasers," Appl. Phys. Lett., vol. 55, pp. 1704-1706, 1989

[19] A. Moritz, R. Wirth, S. Heppel, C. Geng, J. Kuhn, H. Schweizer, F. Scholz, and A. Hangleiter, "Intrinsic modulation bandwidth of strained GaInP/AlGaInP quantum well lasers," Appl. Phys. Lett., vol. 71, pp. 650-652, 1997.

[20] C. B. Su, J. Eom, C. H. Lange, C. B. Kim, R. B. Lauer, W. C. Rideout, and J. S. LaCourse, "Characterization of the dynamics of semiconductor lasers using optical modulation," IEEE J. Quantum Electron., vol. 28, pp. 118-127, 1992.

[21] K. J. Vahala and M. A. Newkirk, "Parasitic-free modulation of semiconductor lasers," IEEE J. Quantum Electron., vol. 25, pp. 1393-1398, 1989.

[22] J. Eom, C. B. Su, J. S. LaCourse, and R. B. Lauer, "The relation of doping level to $\mathrm{K}$ factor and the effect on ultimate modulation performance of semiconductor lasers," IEEE Photon. Technol. Lett., vol. 2, pp. 692-694, 1990.
[23] D. Vassilovski, T. C. Wu, S. Kan, K. Y. Lau, and C. E. Zah, "Unambiguous determination of quantum capture, carrier diffusion, and intrinsic effect in quantum-well laser dynamics using wavelength-selective optical modulation," IEEE Photon. Technol. Lett., vol. 7, pp. 706-708, 1995.

[24] T. Keating, X. Jin, S. L. Chuang, and K. Hess, "Temperature dependence of electrical and optical modulation responses of quantum-well lasers," IEEE J. Quantum Electron., vol. 35, pp. 1526-1534, 1999.

[25] T. Keating, J. Minch, C. S. Chang, P. Enders, W. Fang, S. L. Chuang, T. Tanbun-Ek, Y. K. Chen, and M. Sergent, "Optical gain and refractive index of a laser amplifier in the presence of pump light for cross-gain and cross-phase modulation," IEEE Photon. Technol. Lett., vol. 9, pp. 1358-1360, 1997.

[26] D. A. O. Davies, "Small-signal analysis of wavelength conversion in semiconductor laser amplifiers via gain saturation," IEEE Photon. Technol. Lett., vol. 7, pp. 617-619, 1995.

[27] J. Minch, S. H. Park, T. Keating, and S. L. Chuang, "Theory and experiment of $\mathrm{In}_{1-x} \mathrm{Ga}_{x} \mathrm{As}_{y} \mathrm{P}_{1-y}$ and $\mathrm{In}_{1-x-y} \mathrm{Ga}_{x} \mathrm{Al}_{y}$ As long-wavelength strained quantum-well lasers," IEEE J. Quantum Electron., vol. 35, pp. $1526-1534,1999$.

[28] J. Eom, C. B. Su, J. LaCourse, and R. B. Lauer, "Simultaneous measurement of spontaneous emission rate, nonlinear gain coefficient, and carrier lifetime in semiconductor lasers using a parasitic-free optical modulation technique," Appl. Phys. Lett., vol. 56, pp. 518-520, 1990.

[29] V. Annovazzi-lodi, A. Scire, M. Sorel, and S. Donati, "Dynamic behavior and locking of a semiconductor laser subjected to external injection," IEEE J. Quantum Electron., vol. 34, pp. 2350-2357, 1998.

[30] N. Moore and K. Y. Lau, "Ultrahigh efficiency microwave signal transmission using tandem-contact single quantum well GaAlAs lasers,” Appl. Phys. Lett., vol. 55, pp. 936-938, 1989.

[31] N. Tessler, M. Margalit, R. B. Michael, M. Orenstein, and G. Eisenstein, "Modulation of quantum well lasers by short optical excitation: Energy and spatial dependent effects," IEEE Photon. Technol. Lett., vol. 7, pp. $23-25,1995$.

[32] J. Eom and C. B. Su, "Observation of positive and negative nonlinear gain in an optical injection experiment: Proof of the cavity standingwave-induced nonlinear gain theory in $1.3 \mu \mathrm{m}$ wavelength semiconductor diode lasers," Appl. Phys. Lett., vol. 54, pp. 1734-1736, 1990.

[33] M. Yoshino and K. Inoue, "Improvement of saturation output power in semiconductor laser amplifier through pumping light injection," IEEE Photon. Technol. Lett., vol. 8, pp. 58-59, 1996.

[34] G. R. Gray and G. P. Agrawal, "Effect of cross saturation on frequency fluctuations in a nearly single-mode semiconductor laser," IEEE Photon. Technol. Lett., vol. 3, pp. 204-206, 1991.

[35] M. Ishikawa, R. Nagarajan, T. Fukushima, J. Wasserbauer, and J. Bowers, "Long wavelength high-speed semiconductor laser with carrier transport effects," IEEE J. Quantum Electron., vol. 28, pp. 2230-2241, 1992.

X. Jin was born in Beijing, China, in 1969. She received the B.S. and M.S. degrees in electronic engineering from Tsinghua University, Beijing, China, in 1992 and 1996, respectively. She is currently working toward the Ph.D. degree at the University of Illinois at Urbana-Champaign

Since 1997, she has been conducting research in the Optoelectronics Group, Department Electrical and Computer Engineering, University of Illinois at Urbana-Champaign. Her current research interests are high-speed modulation of semiconductor lasers and modulators.

T. Keating, photograph and biography not available at the time of publication. 
S. L. Chuang received the B.S. degree in electrical engineering from National Taiwan University, Taiwan, in 1976, and the M.S., E.E., and Ph.D. degrees in electrical engineering from the Massachusetts Institute of Technology, Cambridge, in 1980,1981 , and 1983 , respectively.

In 1983, he joined the Department of Electrical and Computer Engineering, University of Illinois at Urbana-Champaign, where he is currently a Professor. He was a Resident Visitor with AT\&T Bell Laboratories, Holmdel, NJ, in 1989, and then a Consultant at Bellcore and Polaroid in 1991. During 1995, he was a Senior Visiting Professor (Sabbatical Chair) at SONY Research Center, and in 1997, an Invited Professor at NTT Basic Research Laboratories. He was also a visitor at NASA Ames Research Center, CA, during the summer of 1999 and an Invited Professor at Fujitsu Research Labs, Japan, in the summer of 2000. He is conducting research on strained quantum-well semiconductor lasers, electroabsorption modulators, fiber optics, and femtosecond nonlinear optics. He is the author of Physics of Optoelectronic Devices (New York: Wiley, 1995) and has published around 200 journal and conference papers. He has given many invited talks at conferences and institutions and has been cited many times for Excellence in Teaching at the University of Illinois.

Dr. Chuang is an Associate Editor of the IEEE JouRnAL OF QUANTUM ELECTRONICS. He was a Feature Editor for the Special Issue on Terahertz Generation, Physics and Applications in the Journal of Optical Society of America B in 1994. He received the Andersen Consulting Award for excellence in advising in 1994 and was selected as an Associate at the Center for Advanced Study at the University of Illinois in 1995. He was also awarded a Fellowship from the Japan Society for the Promotion of Science to visit the University of Tokyo in 1996. He is a Fellow of the Optical Society of America, and a member of the American Physical Society. 